\title{
Simulation of Modes in Terahertz Quantum-Cascade Microring and Microdisk Lasers
}

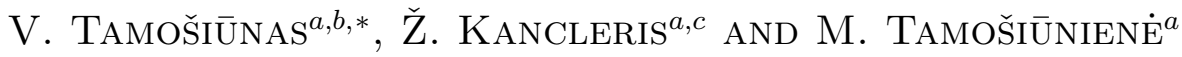 \\ ${ }^{a}$ Semiconductor Physics Institute
}

A. Goštauto 11, LT-01108 Vilnius, Lithuania

${ }^{b}$ Vilnius Gediminas Technical University

Saulètekio al. 11, LT-10223 Vilnius, Lithuania

${ }^{c}$ Faculty of Physics, Vilnius University

Saulètekio al. 9, LT-10222 Vilnius, Lithuania

\begin{abstract}
In our contribution, we report on finite-difference time-domain simulations of modes in microring and microdisk terahertz quantum-cascade lasers with external radii of $100 \mu \mathrm{m}$ and less. Our simulations have revealed a possibility of low radiative loss for lowest and second order whispering gallery modes in such devices, even when the effective mode volume is reduced below the cubic wavelength.
\end{abstract}

PACS numbers: 42.50.Pq, 42.55.Sa, 42.60.Da

\section{Introduction}

One of the most promising types of sources for terahertz range are so-called quantum-cascade lasers (QCLs). These semiconductor heterostructure based devices are compact and relatively easily produced using state of the art semiconductor growth and processing techniques. However, operation of THz QCLs is still restricted to low cryogenic temperatures and the further improvement of their properties, such as operating temperature, duty cycle and threshold current, are strongly dependent on proper design of the heterostructure and waveguide properties of the laser. Therefore, comprehensive investigation of the laser waveguide properties becomes increasingly important, once one tries to improve the laser operation for an already optimized band structure design.

Similar approach was already successfully employed previously in mid-IR devices [1] and in large far-IR devices [2, 3], but the resulting device spectra were

${ }^{*}$ corresponding author; e-mail: vincas@pfi.lt 
harder to analyze due to large number of available modes, so our current investigation would permit a more straightforward comparison with the experimental data.

\section{Simulation method}

Three-dimensional finite-difference time-domain (FDTD) [4] simulations were applied in order to obtain the spectra of the investigated devices. Several additional features, such as two-level gain medium and inversion reduction due to oscillating field increase were incorporated into classical FDTD algorithm as described in more detail in [5] and included references.

$350 \mathrm{ps}$ of wave propagation within the $15.4 \mu \mathrm{m}$ thick "double-metal" type waveguide were simulated. The Yee cell size was set to $1.4 \mu \mathrm{m}$ and Courant number to 0.5 during the simulation. The average relative dielectric constant of GaAs $/ \mathrm{Al}_{0.15} \mathrm{Ga}_{0.85} \mathrm{As}$ medium was set to $\varepsilon=13$. Five single-cycle sources were simulated in order to obtain the initial broad-spectrum waves in different locations of the cavity - one source was placed in the center of the resonator, four others $-2.8 \mu \mathrm{m}$ away from the outer border of the resonator. The amplitudes of sources were varied in order to exclude the enforcement of an additional symmetry. The time-domain data from the last 233 ps of simulated wave propagation was reprocessed in order to obtain the electric field amplitudes corresponding to different frequencies. The maximum of the electric field amplitude near the bottom of the resonator represents a data point of the spectrum for the given frequency.

\section{Simulation results}

The simulation results are presented in Fig. 1. As one can clearly see, three modes with highest maximum electric field exhibit a nearly constant difference between the emission lines in frequency scale. These three modes are the lowest whispering-gallery modes (WGMs) with just one maximum in the radial direction and three different mode numbers $m=4,5,6$, describing the azimuthal variation of the electric field. The fourth mode appears in this device due to a relatively large thickness of the laser. In fact, this thickness exceeds a half of the emission wavelength in the material in the upper part of the investigated frequency range, therefore, waves "bouncing" between the top and the bottom of the resonator become possible.

A different situation occurs in microring resonators. Here $m=0$ mode becomes suppressed, once material is removed from the center of the resonator, however, modes with 2 maxima in radial direction and different numbers $m$ appear, so the resulting spectrum now consists of two overlapping sets of equally spaced modes, as displayed in the middle spectrum of Fig. 1. All three types of modes have favorable conditions to appear in larger microdisks (bottom spectrum of Fig. 1). As one can clearly see, $m=0$ mode is shifted only by approximately $0.2 \mathrm{THz}$ once the outer radius is reduced from $r_{\text {out }}=50 \mu \mathrm{m}$ to $r_{\text {out }}=35 \mu \mathrm{m}$. Such small 


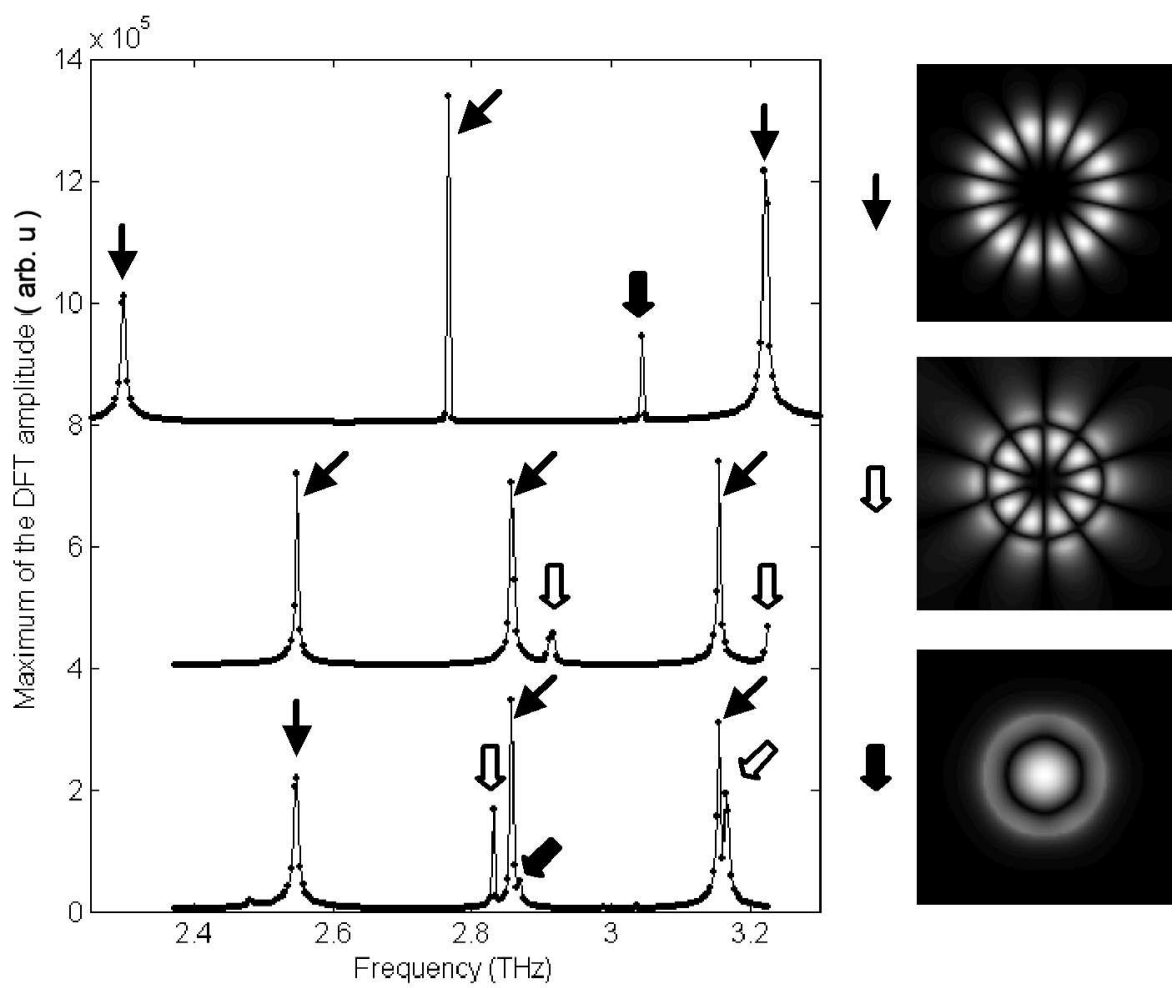

Fig. 1. (left) Simulated spectra of terahertz microring and microdisk lasers: (top) microdisk with $r=35 \mu \mathrm{m}$ radius, (middle) microring with $r_{\text {out }}=50 \mu \mathrm{m}$ outer and $r_{\text {in }}=25 \mu \mathrm{m}$ inner radii, and (bottom) microdisk with $r=50 \mu \mathrm{m}$ radius. Spectra are shifted in vertical axis for clarity. (right) Typical distributions of electric field near the bottom of the resonator for 3 types of modes indicated with corresponding arrows.

reduction occurs due to the proximity of the cut-off frequency. In such situation, the effective wave number for this mode is mainly defined by the thickness, while dimensions in the radial direction have much smaller influence. Also, one more important feature becomes clear once comparing the spectra of microdisk and microring resonators - the removal of material from the center of the resonator influences emission lines of the second order modes substantially more than lines of lowest WGMs with positions of the later ones remaining nearly constant. This effect might be employed when designing future devices with double line emission spectrum and defined frequency difference between them.

\section{Conclusions}

Our numerical investigations of terahertz quantum-cascade laser microcavities revealed that three types of modes might be observed in case of microdisks with $r=50 \mu \mathrm{m}$ radius and gain maximum at $2.8 \mathrm{THz}$. In microring configuration, 
only lowest and second order WGMs remain possible, with much larger emission frequency shifts in case of the second order modes. The second order modes are suppressed once radius of the microdisk is reduced to $r=35 \mu \mathrm{m}$.

\section{Acknowledgements}

This work was supported, in part, by Lithuanian State Science and Studies Fundation contract No C-07004.

\section{References}

[1] S. Anders, V. Tamosiunas, W. Schrenk, G. Strasser, Phys. Rev. B 69, 073309 (2004).

[2] G. Fasching, A. Benz, R. Zobl, A.M. Andrews, T. Roch, W. Schrenk, G. Strasser, V. Tamosiunas, K. Unterrainer, Proc. SPIE Int. Soc. Opt. Eng. 6010, 601006 (2005).

[3] G. Fasching, A. Benz, R. Zobl, A.M. Andrews, T. Roch, W. Schrenk, G. Strasser, V. Tamosiunas, K. Unterrainer, Physica E, Low-Dimens. Syst. Nanostruct. 32, 316 (2006).

[4] A. Taflove, Computational Electrodynamics: The Finite-Difference Time-Domain Method, Artech House, Norwood, MA 1995.

[5] V. Tamošiūnas, Ž. Kancleris, M. Dagys, R. Simniškis, M. Tamošiūnienè, G. Valušis, G. Strasser, K. Unterrainer, Acta Phys. Pol. A 107, 179 (2005). 